\title{
Clinical Study \\ Extended High Frequency Audiometry in Polycystic Ovary Syndrome
}

\author{
Cuneyt Kucur, ${ }^{1}$ Suna Kabil Kucur, ${ }^{2}$ Ilay Gozukara, ${ }^{2}$ Ali Seven, \\ Kadriye Beril Yuksel, ${ }^{2}$ Nadi Keskin, ${ }^{2}$ and Fatih Oghan ${ }^{1}$ \\ ${ }^{1}$ Medical School, Evliya Celebi Training and Research Hospital, Department of Otolaryngology, Dumlupinar University, \\ Okmeydani Street, 43340 Kutahya, Turkey \\ ${ }^{2}$ Department of Obstetrics and Gynecology, Medical School, Dumlupinar University, 43340 Kutahya, Turkey
}

Correspondence should be addressed to Cuneyt Kucur; ckucur@yahoo.com.tr

Received 7 June 2013; Accepted 26 August 2013

Academic Editors: H. Hiraumi and A. Teymoortash

Copyright (C) 2013 Cuneyt Kucur et al. This is an open access article distributed under the Creative Commons Attribution License, which permits unrestricted use, distribution, and reproduction in any medium, provided the original work is properly cited.

\begin{abstract}
Objective. Polycystic ovarian syndrome (PCOS) is the most common endocrine disorder affecting 5-10\% of women in reproductive age. Insulin resistance, dyslipidemia, glucose intolerance, hypertension, and obesity are metabolic disorders accompanying the syndrome. PCOS is a chronic proinflammatory state and the disease is associated with endothelial dysfunction. In diseases with endothelial damage, hearing in high frequencies are mostly effected in early stages. We evaluated extended high frequency hearing loss in PCOS patients. Material Methods. Forty women diagnosed as PCOS and 25 healthy controls were included in this study. Age and BMI of PCOS and control groups were comparable. Each subject was tested with low $(250-2000 \mathrm{~Hz})$, high $(4000-8000 \mathrm{~Hz})$, and extended high frequency audiometry (8000-20000). Hormonal and biochemical values including LH, LH/FSH, testosterone, fasting glucose, fasting insulin, HOMA-I, and CRP were calculated. Results. PCOS patients showed high levels of LH, LH/FSH, testosterone, fasting insulin, glucose, HOMA-I, and CRP levels. The hearing thresholds of the groups were similar at frequencies of 250,500, 1000, 2000, and $4000 \mathrm{~Hz}$; statistically significant difference was observed in $8000-14000 \mathrm{~Hz}$ in PCOS group compared to control group. Conclusion. PCOS patients have hearing impairment especially in extended high frequencies. Further studies are needed to help elucidate the mechanism behind hearing impairment in association with PCOS.
\end{abstract}

\section{Introduction}

Polycystic ovarian syndrome (PCOS) is the most common endocrine disorder affecting $5-10 \%$ of women in reproductive age [1]. The etiology is unknown. Disease is characterized by oligo-amenorrhea, hyperandrogenism, and polycystic ovaries [2]. PCOS is a chronic condition beginning most commonly in adolescence. Insulin resistance, dyslipidemia, glucose intolerance, hypertension, obesity, and nonalcoholic fatty liver are metabolic disorders accompanying syndrome [3-5]. Therefore, a higher cardiovascular risk is reported [6]. Endothelial cell dysfunction is one of the earliest stages of atherogenesis. Therefore, markers reflecting endothelial injury have been searched [7]. Tumor necrosis factor, highly sensitive C-Reactive Protein, homocysteine, and Plasminogen activator inhibitor-1 are some of the cardiovascular risk markers that are increased in PCOS [8]. PCOS is a chronic proinflammatory state. An imbalance between prooxidants and antioxidants in PCOS produces an oxidative state [9]. There is also an association between inflammation at the molecular level and insulin resistance in the disorder $[8,10]$. Elevations of a number of circulating proatherogenic inflammatory mediators have been independently reported in PCOS [11, 12]. Meta-analysis of the 31 articles reported that circulating CRP was 96\% higher in women with PCOS compared to healthy controls [13]. The relationship between CRP and atherothrombotic cardiovascular disease, renal function abnormalities, has been reported in a number of studies [14]. Factors predisposing for endothelial injury include hyperinsulinemia, insulin resistance, dyslipidemia, and chronic lowgrade inflammation which often accompanies PCOS [15].

Recently, a study conducted by Oghan et al. showed that patients with PCOS have sensorineural hearing loss in high frequencies [16]. They postulated that hyperandrogenism 
was the possible etiological factor. However, to date, the relationship between PCOS and hearing has not been fully understood. The aim of the present study was to determine the status of extended high frequency audiometry in cases of PCOS and to evaluate if there are other possible contributing factors that cause hearing loss.

\section{Material Method}

The study was carried out at Dumlupinar University Kutahya Evliya Celebi Training and Research Hospital gynecology outpatient clinic. Forty patients with the PCOS and twentyfive healthy subjects were enrolled in this prospective study. The control group consisted of patients who admitted to hospital for routine gynecological examination.

We included healthy women as controls with normal menstrual cycles, with no evidence of hyperandrogenism, and with normal ovarian morphology on pelvic ultrasonography. Ferriman Gallwey scores of all control patients were under 8. PCOS was defined as the presence of two of the following three features after the exclusion of other etiologies [17], (i) oligo- or anovulation (fewer than six menstrual periods in the preceding year), (ii) hyperandrogenism and/or biochemical signs of hyperandrogenism, and/or (iii) polycystic ovaries.

The study was conducted according to the guidelines for clinical studies described in the Declaration of Helsinki (as revised by the World Medical Association, http://www.wma.net/). Regional Ethical Committee approved the study. All patients gave oral and written informed consent prior to the examination.

Exclusion criteria were tinnitus, middle ear disease, diabetes mellitus, family history of hearing loss, history of acoustic trauma, conductive hearing loss, exposure to ototoxic substances, occupational noise exposure, autoimmune diseases, history of smoking, ongoing infection-inflammation, and being on any medication.

Body mass index (BMI) was calculated as weight (kg)/height $(\mathrm{m})^{2}$. Systolic (SBP) and diastolic blood pressure (DBP) were measured twice in the right arm in a relaxed sitting position. Two measurements were taken 15 minutes apart. The average of two was used.

Blood samples were collected during early follicular phase of menstrual cycle after at least 12-hour fasting. Levels of glucose, insulin, and hormone profile (follicle-stimulating hormone (FSH), luteinizing hormone (LH), estradiol (E2), total and free testosterone (Total-T and Free-T), and prolactin $(\mathrm{PRL}))$ were determined. Plasma glucose was determined with the glucose hexokinase method (Cobas Integra 400 Plus, Roche Diagnostics, and Mannheim, Germany). Hormone profile is measured with electrochemiluminescence assays (ELECYS 2010 HITACHI, Roche Diagnostic, Germany).

Plasma concentrations of insulin were measured by chemi-luminescent immunoassay (Immulite One; BioDPC, Los Angeles, CA, USA). Insulin resistance was measured with HOMA-IR (homeostasis model assessment for insulin resistance) [18].

All the participants were subjected to careful ear examination to identify any abnormalities that may interfere with
TABLE 1: Demographic findings of subjects.

\begin{tabular}{lcccc}
\hline Groups & $N$ & Mean & Standard deviation & $P$ \\
\hline \multirow{2}{*}{ Age control PCOS } & 25 & 24,6400 & 4,64471 & 0,465 \\
& 40 & 23,7843 & 4,83865 & \\
\multirow{3}{*}{ BMI control PCOS } & 25 & 24,3960 & 3,81930 & 0,155 \\
& 40 & 25,9784 & 4,80293 & \\
\hline
\end{tabular}

TABLE 2: Biochemical parameters of PCOS and control group.

\begin{tabular}{lccc}
\hline & PCOS & Control & $P$ \\
\hline Fasting Glucose & $85,2 \pm 29,8$ & $82,7 \pm 37,3$ & $\mathbf{0 , 0 2 7}$ \\
Insulin & $14,3 \pm 7,8$ & $4,9 \pm 1,2$ & $\mathbf{0 , 0 2 8}$ \\
Homa-1 & $3,2 \pm 2,7$ & $1,6 \pm 0,3$ & $\mathbf{0 , 0 2 0}$ \\
CRP & $0,3 \pm 0,4$ & $0,1 \pm 0,1$ & $\mathbf{0 , 0 1 6}$ \\
MPV & $8,3 \pm 0,9$ & $8,1 \pm 1,0$ & 0,644 \\
TSH & $2,3 \pm 1,1$ & $2,2 \pm 1,1$ & 0,859 \\
LH & $9,5 \pm 5,5$ & $5,7 \pm 2,7$ & $\mathbf{0 , 0 0 4}$ \\
FSH & $5,1 \pm 2,2$ & $4,0 \pm 1,9$ & $\mathbf{0 , 0 3 9}$ \\
LH/FSH & $2,0 \pm 1,1$ & $1,4 \pm 0,3$ & $\mathbf{0 , 0 4 5}$ \\
E2 & $96,9 \pm 78,5$ & $111,0 \pm 97,6$ & 0,324 \\
Progesterone & $5,3 \pm 9,4$ & $7,5 \pm 7,5$ & 0,494 \\
PRL & $15,5 \pm 7,9$ & $17,2 \pm 8,4$ & 0,403 \\
Testosterone & $0,4 \pm 0,3$ & $0,3 \pm 0,1$ & $<\mathbf{0 , 0 0 0 1}$ \\
\hline
\end{tabular}

hearing such as a perforated tympanic membrane or other middle ear pathologies. All subjects had normal immittance audiometry results. Each participant was tested with Low Frequency Audiometry (LFA); $125 \mathrm{~Hz}$ to $2 \mathrm{kHz}$, High Frequency Audiometry (HFA); $4 \mathrm{kHz}$ to $8 \mathrm{kHz}$, and Extended High Frequency Audiometry (EHFA); $9 \mathrm{kHz}$ to $20 \mathrm{kHz}$. Audiometry was performed by an expert audiologist blinded to the study.

All statistical analyses were performed using the SPSS for Windows, version 17.0. Nonparametric tests were used as the variable hearing threshold had abnormal distribution due to data dispersion and lack of distribution symmetry. After testing the normal distribution, comparisons between the groups were tested using the $t$-test. The chosen significance level was $P<0.05$.

\section{Results}

Following through examination, otologic and audiometric evaluation, 40 patients with PCOS and 25 healthy controls were included in the final analysis. Two groups were comparable with regard to age and BMI. The mean age was $23.8 \pm 4.6$ years in PCOS group and $24.6 \pm 4.8$ years in control group. The mean BMIs were $25.9 \pm 4.8 \mathrm{~kg} / \mathrm{m}^{2}$ and $24.4 \pm$ 3.8. Demographical and laboratory findings are shown in Tables 1 and 2 .

A significant difference in LH, LH/FSH, Testosterone, CRP, fasting glucose, fasting insulin, and HOMA index was observed among the two groups (Table 2).

The hearing thresholds for the left and right ears are described in Table 3. Although the hearing thresholds of the 
TABLE 3: Hearing thresholds of PCOS and control group.

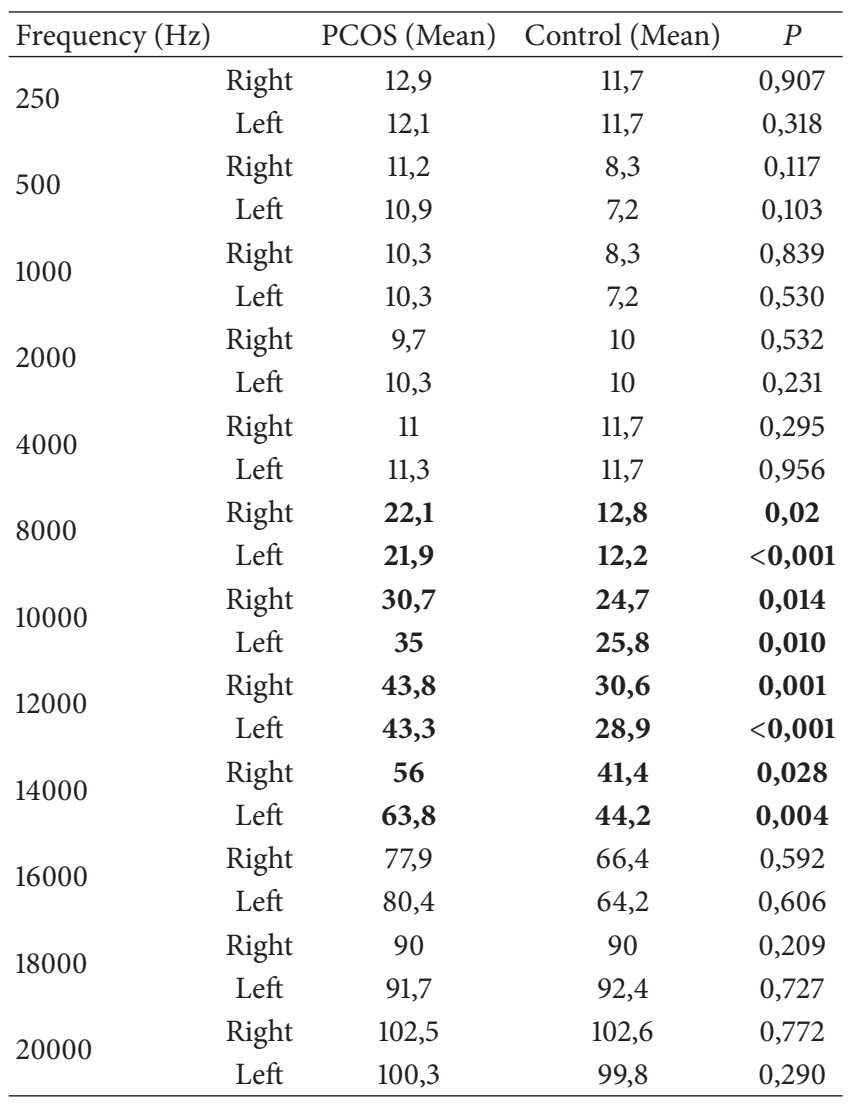

groups were similar at frequencies of 250, 500, 1000, 2000, and $4000 \mathrm{~Hz}$, significant hearing loss was observed at frequencies of $8000,10000,12000$, and $14000 \mathrm{~Hz}$ in PCOS group compared to controls (Figure 1). There was no statistically significant difference in tympanometric results between the two groups.

\section{Discussion}

To the best of our knowledge, this is the first study evaluating extended high frequency hearing loss in women with PCOS compared to healthy women. Routine audiometry is restricted to $125-8000 \mathrm{~Hz}$ frequencies. However, in diseases with endothelial damage, high frequencies are mostly affected in early stages. High frequency audiometry was introduced into clinical practice in 1960s [19].

In the present study, HOMA-I, CRP, LH, LH/FSH, testosterone, fasting glucose, and insulin levels were higher in women with PCOS. CRP is a circulating marker of the proinflammatory state in PCOS as evidenced by the 2-fold elevation in circulating CRP compared to controls. Similarly, in our study CRP was significantly higher in PCOS patients $(P<0.05)$. A meta-analysis of the most comparable studies indicates that circulating CRP is elevated in PCOS reflective of the chronic low-grade inflammation present in the disorder. They also found that elevated circulating CRP in PCOS is independent of obesity [13]. Although we could not demonstrate a direct correlation between CRP levels and

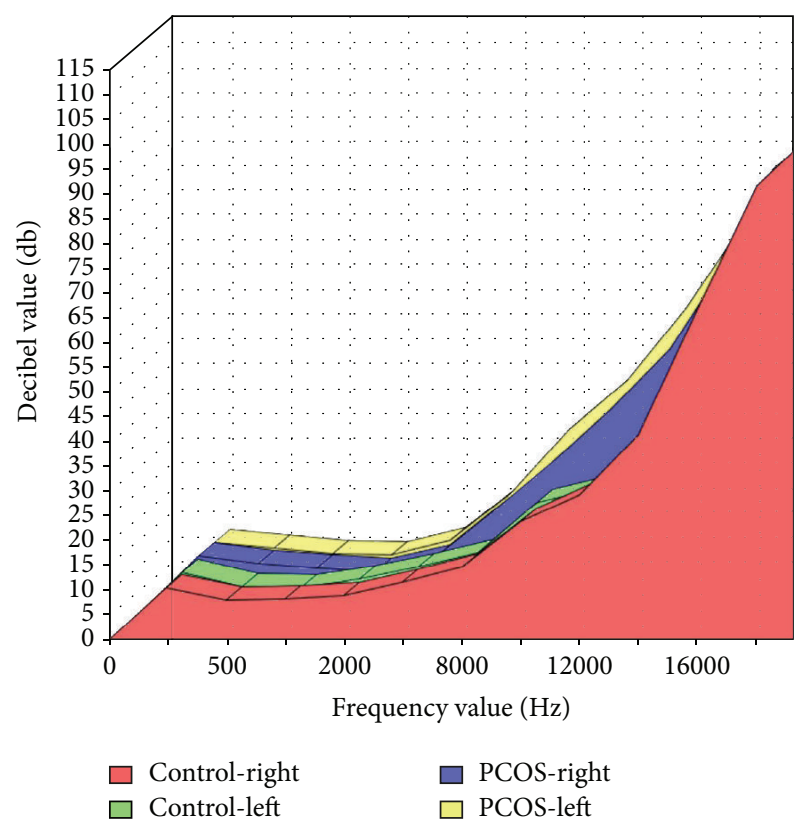

FIGURE 1: Comparison of hearing levels between groups.

hearing thresholds, chronic inflammation may play a role in the pathogenesis of hearing impairment in PCOS. Main causes of hearing loss seen in EHFA in PCOS patients may also be multifactorial.

Early signs of endothelial damage and increased cardiovascular disease risk have been previously described in PCOS patients [20]. Atherogenesis and endothelial dysfunction have been attributed to a number of biochemical and hormonal alterations in PCOS. Elevated mean platelet volume, white blood cell, D-Dimer, and androgen levels have been reported as potential indicators of risk factor for atherogenesis $[21,22]$.

EHFA with determination of air conduction thresholds in the frequency range of $8-20 \mathrm{kHz}$ has been used in a number of studies in recent years [23]. Sensorineural hearing loss has been reported in a number of chronic systemic inflammatory disorders such as ankylosing spondylitis and rheumatoid arthritis, but exact cause of underlying pathology is still unknown [24, 25]. However, immunomediated vasculitis in the inner ear and ototoxic medication used for treatment was postulated as causative agents in some. Hearing losses in autoimmune diseases are reported to be mediated by vascular mechanisms [26].

Oghan and Coksuer firstly described high frequency $(4000-8000 \mathrm{~Hz})$ hearing loss in PCOS patients. They attributed this to the hyperandrogenism seen in the syndrome [16]. In the present study, we evaluated the hearing thresholds in EHF (8000-20000 Hz). We observed statistically significant difference in hearing thresholds between PCOS and control groups in $8000,10000,12000$, and $14000 \mathrm{~Hz}$. There were no significant differences in hearing thresholds comparing right to left ears at all frequencies $(250-20000 \mathrm{~Hz})$.

Despite certain limitations of our study, the results might be as preliminary findings for designing future studies on 
larger populations. A limitation of this study is small population, but strength of this study is evaluating the EHFA in all participants.

High frequencies are more sensitive to vascular damage caused by the disease. Insulin resistance, hyperandrogenemia, and elevated serum CRP as an inflammatory marker in PCOS could be the cause of hearing loss in especially extended high frequencies. Our data suggest that HFA and EHFA are more efficient in detecting early hearing loss compared to pure tone audiometry. Further studies are needed to help elucidate the mechanism behind hearing impairment in association with PCOS and to see whether the impairment of EHFA in these cases are progressive. It might be possible to prevent progression of hearing impairment by revealing underlying factors.

\section{References}

[1] R. Azziz, K. S. Woods, R. Reyna, T. J. Key, E. S. Knochenhauer, and B. O. Yildiz, "The prevalence and features of the polycystic ovary syndrome in an unselected population," Journal of Clinical Endocrinology and Metabolism, vol. 89, no. 6, pp. 2745-2749, 2004.

[2] B. C. J. M. Fauser, "Revised 2003 consensus on diagnostic criteria and long-term health risks related to polycystic ovary syndrome," Fertility and Sterility, vol. 81, no. 1, pp. 19-25, 2004.

[3] K. A. Toulis, D. G. Goulis, G. Mintziori et al., "Meta-analysis of cardiovascular disease risk markers in women with polycystic ovary syndrome," Human Reproduction Update, vol. 17, no. 6, pp. 741-760, 2011.

[4] C. M. DeUgarte, A. A. Bartolucci, and R. Azziz, "Prevalence of insulin resistance in the polycystic ovary syndrome using the homeostasis model assessment," Fertility and Sterility, vol. 83, no. 5, pp. 1454-1460, 2005.

[5] T. L. Setji, N. D. Holland, L. L. Sanders, K. C. Pereira, A. M. Diehl, and A. J. Brown, "Nonalcoholic steatohepatitis and nonalcoholic fatty liver disease in young women with polycystic ovary syndrome," Journal of Clinical Endocrinology and Metabolism, vol. 91, no. 5, pp. 1741-1747, 2006.

[6] T. Cascella, S. Palomba, I. De Sio et al., "Visceral fat is associated with cardiovascular risk in women with polycystic ovary syndrome," Human Reproduction, vol. 23, no. 1, pp. 153159, 2008.

[7] G. S. Caglar, E. Oztas, D. Karadag, R. Pabuccu, and A. A. Eren, "The association of urinary albumin excretion and metabolic complications in polycystic ovary syndrome," European Journal of Obstetrics Gynecology \& Reproductive Biology, vol. 154, no. 1, pp. 57-61, 2011.

[8] F. González, N. S. Rote, J. Minium, and J. P. Kirwan, "Increased activation of nuclear factor $\kappa \mathrm{B}$ triggers inflammation and insulin resistance in polycystic ovary syndrome," Journal of Clinical Endocrinology and Metabolism, vol. 91, no. 4, pp. 15081512, 2006.

[9] J. R. Palacio, A. Iborra, Z. Ulcova-Gallova, R. Badia, and P. Martínez, "The presence of antibodies to oxidative modified proteins in serum from polycystic ovary syndrome patients," Clinical and Experimental Immunology, vol. 144, no. 2, pp. 217222, 2006.
[10] F. González, N. S. Rote, J. Minium, and J. P. Kirwan, "In vitro evidence that hyperglycemia stimulates tumor necrosis factor$\alpha$ release in obese women with polycystic ovary syndrome," Journal of Endocrinology, vol. 188, no. 3, pp. 521-529, 2006.

[11] E. Diamanti-Kandarakis, T. Paterakis, K. Alexandraki et al., "Indices of low-grade chronic inflammation in polycystic ovary syndrome and the beneficial effect of metformin," Human Reproduction, vol. 21, no. 6, pp. 1426-1431, 2006.

[12] W.-H. Hu, J. Qiao, S.-Y. Zhao, X.-W. Zhang, and M.-Z. Li, "Monocyte chemoattractant protein-1 and its correlation with lipoprotein in polycystic ovary syndrome," Beijing Da Xue Xue Bao, vol. 38, no. 5, pp. 487-491, 2006.

[13] F. Héctor, E. Morreale, M. L. Ramírez, and F. González, "Circulating inflammatory markers in polycystic ovary syndrome: a systematic review and metaanalysis," Fertility and Sterility, vol. 95, pp. 1048.e2-1058.e2, 2011.

[14] E. M. Stuveling, H. L. Hillege, S. J. L. Bakker, R. O. B. Gans, P. E. de Jong, and D. de Zeeuw, "C-reactive protein is associated with renal function abnormalities in a non-diabetic population," Kidney International, vol. 63, no. 2, pp. 654-661, 2003.

[15] W. Foltyn, J. Strzelczyk, B. Marek et al., "Selected markers of endothelial dysfunction in women with polycystic ovary syndrome," Endokrynologia Polska, vol. 62, no. 3, pp. 243-248, 2011.

[16] F. Oghan and H. Coksuer, "Does hyperandrogenism have an effect on hearing loss in patients with polycystic ovary syndrome?" Auris Nasus Larynx, vol. 39, no. 4, pp. 365-368, 2012.

[17] B. C. J. M. Fauser, "Revised 2003 consensus on diagnostic criteria and long-term health risks related to polycystic ovary syndrome," Fertility and Sterility, vol. 81, no. 1, pp. 19-25, 2004.

[18] D. R. Matthews, J. P. Hosker, and A. S. Rudenski, "Homeostasis model assessment: insulin resistance and $\beta$-cell function from fasting plasma glucose and insulin concentrations in man," Diabetologia, vol. 28, no. 7, pp. 412-419, 1985.

[19] S. Okstad, I. W. S. Mair, and E. Laukli, "High-frequency audiometry: air-and electric bone-conduction," Acta OtoLaryngologica, Supplement, vol. 106, no. 449, pp. 159-160, 1988.

[20] F. Orio, S. Palomba, and A. Colao, "Cardiovascular risk in women with polycystic ovary syndrome," Fertility and Sterility, vol. 86, no. 1, pp. S20-S21, 2006.

[21] L. Kebapcilar, C. E. Taner, A. G. Kebapcilar, and I. Sari, "High mean platelet volume, low-grade systemic coagulation and fibrinolytic activation are associated with androgen and insulin levels in polycystic ovary syndrome," Archives of Gynecology and Obstetrics, vol. 280, no. 2, pp. 187-193, 2009.

[22] J. Danesh, R. Collins, P. Appleby, and R. Peto, "Association of fibrinogen, C-reactive protein, albumin, or leukocyte count with coronary heart disease: meta-analyses of prospective studies," Journal of the American Medical Association, vol. 279, no. 18, pp. 1477-1482, 1998.

[23] D. Sharma, S. K. Munjal, and N. K. Panda, "Extended high frequency audiometry in secretory otitis media," Indian Journal of Otolaryngology and Head and Neck Surgery, vol. 64, no. 2, pp. 145-149, 2012.

[24] O. K. Kahveci, U. S. Demirdal, A. Duran, A. Altuntas, V. Kavuncu, and E. Okur, "Hearing and cochlear function of patients with ankylosing spondylitis," Clinical Rheumatology, vol. 31, pp. 1103-1108, 2012. 
[25] A. Oztürk, S. Yalçin, I. Kaygusuz et al., "High-frequency hearing loss and middle ear involvement in rheumatoid arthritis," American Journal of Otolaryngology, vol. 25, no. 6, pp. 411-417, 2004.

[26] M. J. Ruckenstein, "Autoimmune inner ear disease," Current Opinion in Otolaryngology \& Head and Neck Surgery, vol. 12, no. 5, pp. 426-430, 2004. 


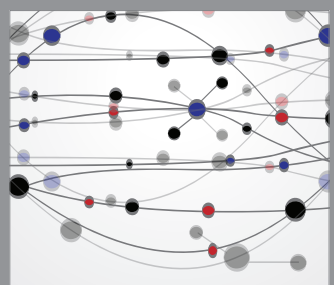

The Scientific World Journal
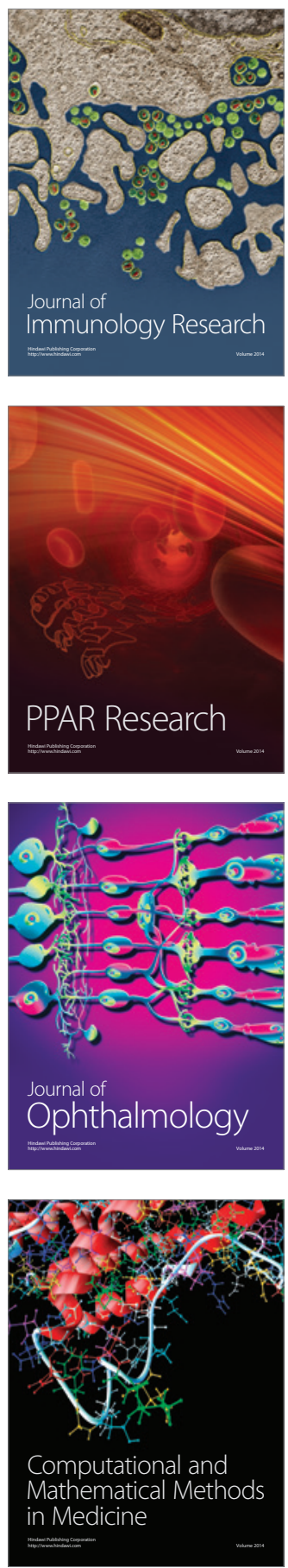

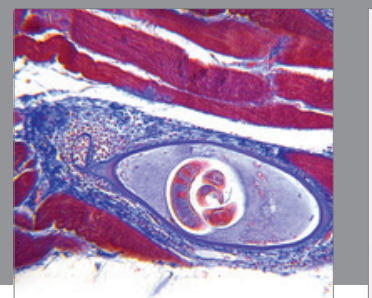

Gastroenterology

Research and Practice
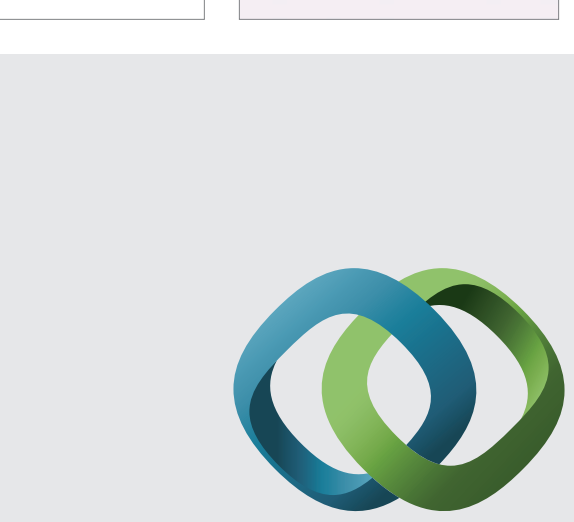

\section{Hindawi}

Submit your manuscripts at

http://www.hindawi.com
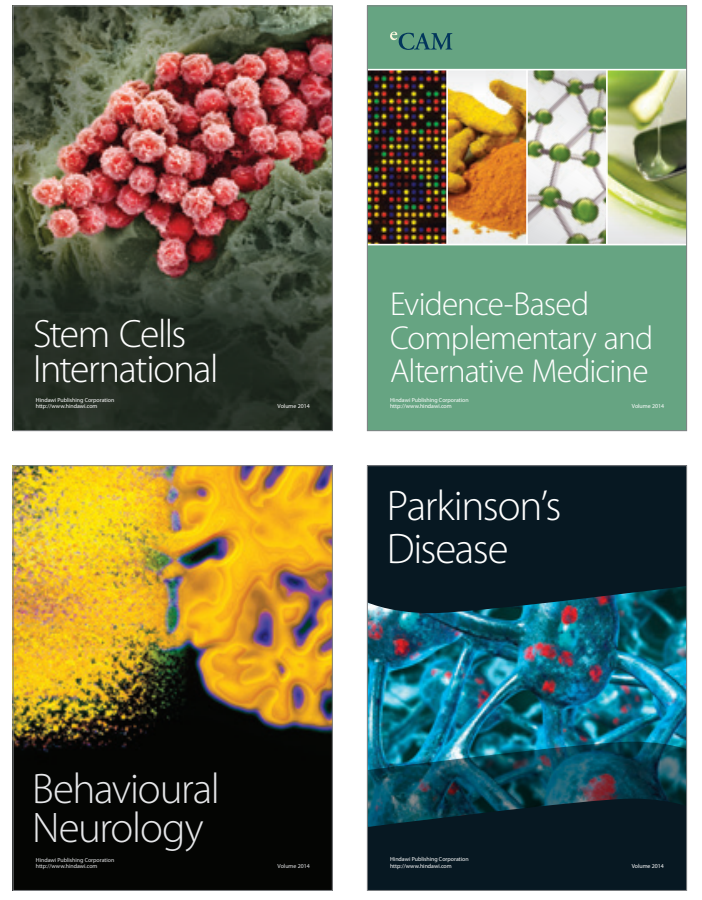
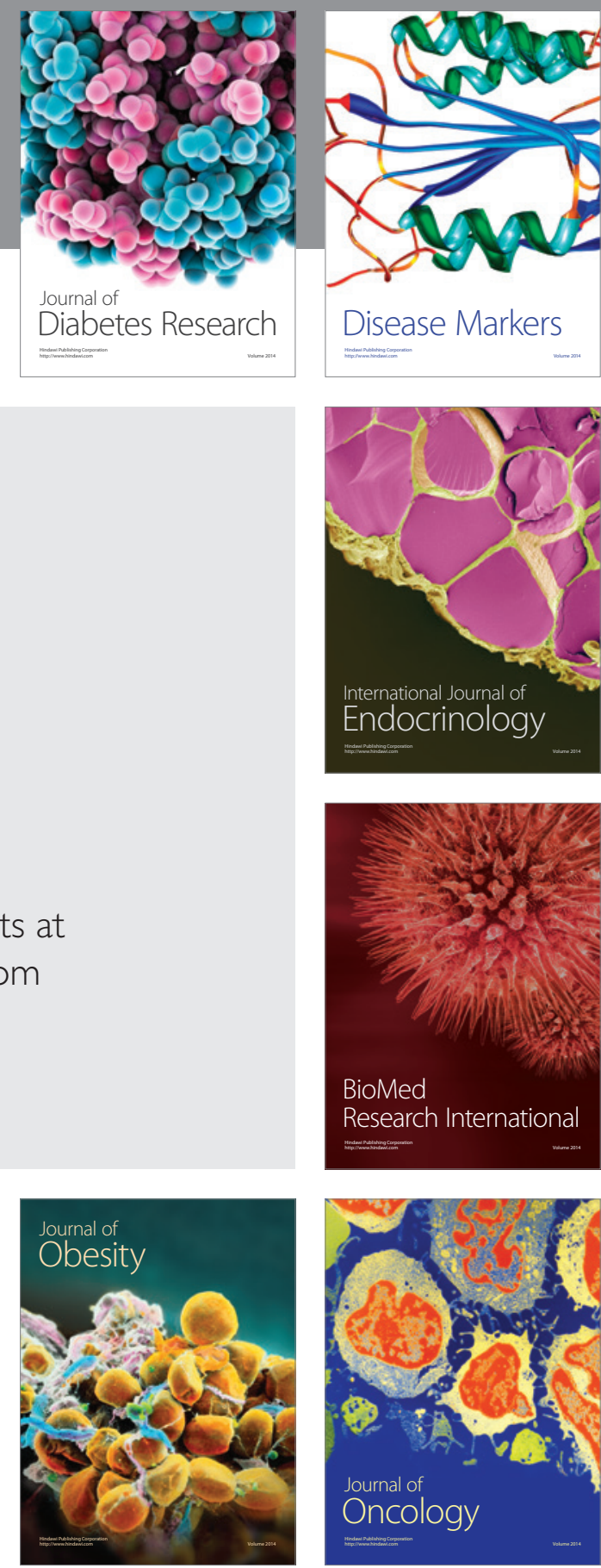

Disease Markers
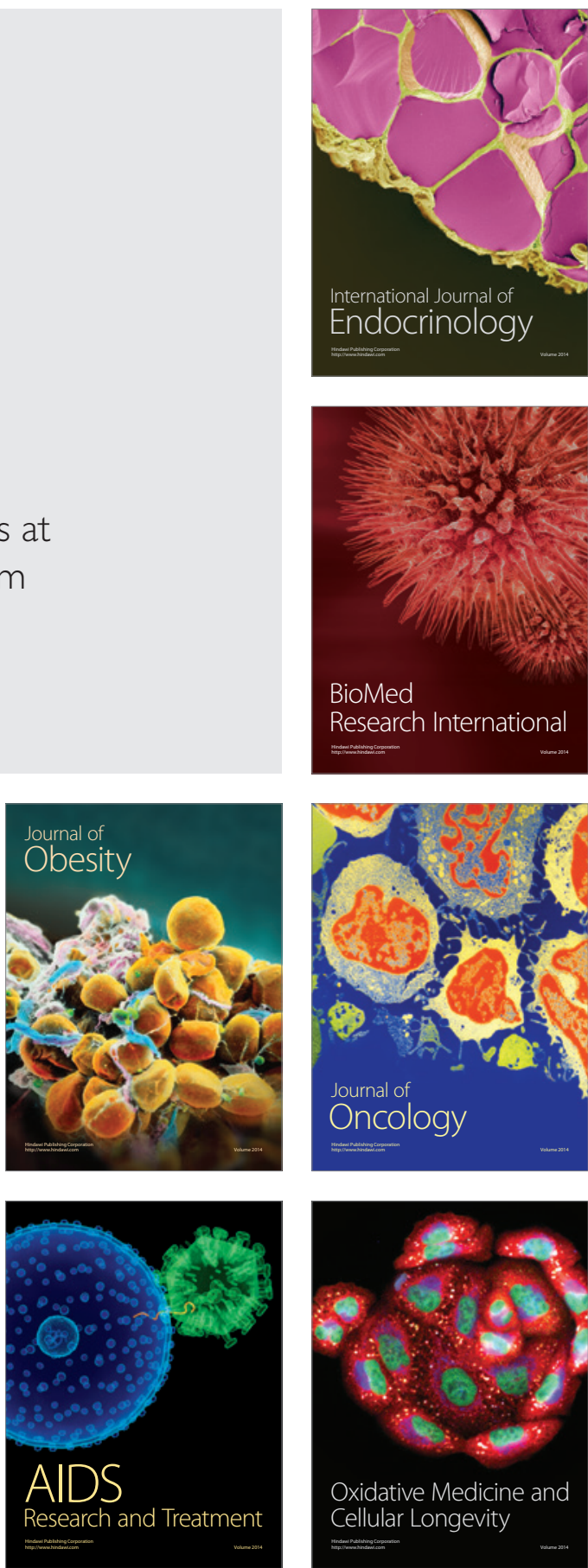